D.O.I.: $10.3895 / \mathrm{S} 1808-04482007000400003$

\title{
ALINHAMENTO ENTRE AS ESTRATÉGIAS COMPETITIVA E DE MANUFATURA: ESTUDOS DE MÚLTIPLOS CASOS NA INDÚSTRIA CALÇADISTA
}

\section{ALIGMENT BETEWEEN COMPETITIVE AND OPERATIONS STRATEGIES: MULTIPLE CASE STUDIES IN FOOTWEAR INDUSTRY}

\author{
Sergio Evangelista Silva ${ }^{1}$; Flávio César Faria Fernandes ${ }^{2}$ \\ ${ }^{1}$ Universidade do Estado de Minas Gerais Campus de Passos FESP/UEMG - Passos - Brasil \\ silvaevangelista@passosuemg.br \\ ${ }^{2}$ Universidade Federal de São Carlos - UFSCar - São Carlos - Brasil \\ dfcf@power.ufscar.br
}

\begin{abstract}
Resumo
Este artigo tem como propósito analisar a relação existente entre aspectos relativos às estratégias competitiva e de manufatura em empresas da indústria calçadista. Foram pesquisadas três empresas de grande porte (que possuem mais de 500 funcionários) situadas na cidade de Franca, interior de São Paulo. Os resultados mostram que a definição da estratégia competitiva possui papel determinante, sobretudo, na configuração das funções marketing e P\&D. Já no que diz respeito à função manufatura, uma determinada estratégia competitiva não implica necessariamente numa única maneira de configurar esta função. Outro aspecto, é que mesmo independente da estratégia escolhida, as empresas sempre dão atenção ao baixo custo de produção.
\end{abstract}

Palavras-chave: estratégia competitiva, estratégia de manufatura, indústria calçadista.

\section{Introdução}

A indústria calçadista é um ramo de atividade em que se encontra alto nível de pressão competitiva entre seus agentes. Os principais fatores que provavelmente contribuem para esta situação no Brasil são: a fabricação do calçado possui caráter inerentemente artesanal, ou seja, esta é uma atividade, sobretudo, dependente de mão-de-obra, e que a maior parte dos equipamentos aqui utilizados são de controle mecânico. Desta forma, a abertura de uma nova manufatura calçadista exige de seus empreendedores pequenos investimentos, se comparada com a abertura de empresas em outros ramos. Estas características são provavelmente os principais motivos para a existência no Brasil de aproximadamente 8000 manufaturas no início desta década, segundo relatório da Abicalçados (ABICALÇADOS, 2003).

As características citadas acima possivelmente colaboraram para as profundas 
transformações que ocorreram na indústria calçadista a partir da segunda metade do século XX. Autores como Anderson (2001) e Schmitz (1999) afirmam que são duas as fases da globalização desta indústria. A primeira ocorreu a partir dos anos 70, quando a fabricação de calçados foi transferida de países desenvolvidos para países em desenvolvimento. Anderson (2001) afirma que nesta ocasião as operações migraram principalmente para o Brasil e tigres asiáticos - Coréia do Sul, Hong Kong e Taiwan. O principal fator desta transferência foi a atratividade dos baixos salários destes países. Não obstante, segundo Schmitz (1999) e Anderson (2001) a indústria calçadista passou ainda por uma segunda fase de transformação a partir da década de 80 , quando ocorreu uma nova transferência das atividades produtivas para países com níveis salariais ainda mais baixos do que aqueles que participaram da primeira fase. São citados como novos centros de produção calçadista: China, Filipinas, Indonésia e Tailândia.

O ambiente de extrema competição da indústria calçadista exige, portanto, de suas empresas a contínua busca do aumento da competitividade, o que se imagina será favorecida por um processo de planejamento deliberado. Ou seja, acredita-se que o aumento da competitividade das empresas nesta indústria esteja baseado na adequada organização e coordenação das várias funções empresariais, o que exige a elaboração de uma estratégia competitiva. Esta estratégia deve ser suportada pelas estratégias em nível funcional como atestam Hayes \& Wheelwright (1984) e Sun e Hong (2002). Neste contexto dá-se destaque à estratégia de manufatura, que tem como propósito guiar as ações desta função e definir como ela dará suporte à estratégia competitiva.

O objetivo deste artigo é analisar como a estratégia de manufatura dá suporte à estratégia competitiva em manufaturas de calçados de grande porte (que possuem mais de 500 funcionários foi utilizada a classificação do SEBRAE, 2007). Nas seções seguintes é realizada: uma revisão da literatura sobre estratégia competitiva e estratégia de manufatura - seção 2 ; a apresentação da metodologia - seção 3; a apresentação dos casos - seção 4; a análise de resultados - seção 5; a elaboração das conclusões - seção 6; e a apresentação das referências bibliográficas - seção 7.

\section{Uma revisão sobre estratégia competitiva e estratégia de manufatura}

\subsection{Estratégia competitiva}

O conceito de estratégia competitiva tem sido amplamente discutido na literatura a partir de várias perspectivas e visões. Pode-se dizer que a grande maioria do público associa ao conceito de estratégia a idéia de planejamento deliberado das atividades de uma empresa, que visam aumentar o seu desempenho no longo prazo. Esta é o que pode ser chamada de visão ideal do conceito de estratégia, que foi primeiramente estabelecida por Igor Ansoff em 1965 em seu livro Corporate Strategy, neste livro este autor lança os conceitos básicos da chamada Escola Clássica. A premissa 
essencial desta escola é que o bom desempenho da empresa depende de um planejamento deliberado.

Autores como Gavetti \& Levinthal (2004), Mintzberg et al (2000) e Whittington (2002) identificaram posteriormente à escola clássica, várias outras escolas de pensamento estratégico, que se dedicam a aprofundar os conceitos desenvolvidos por esta escola, ou em verificar como a estratégia ocorre na realidade.

Dentre as várias escolas identificadas merece destaque aquela que Mintzberg et al (2000) chama de escola de posicionamento, sobretudo o seu principal expoente, Michael Porter, que em seu livro Estratégia Competitiva (PORTER, 1986) identificou três tipos genéricos de estratégias praticados pelas empresas no mercado. São elas: a estratégia de baixo custo, que é quando a empresa busca continuamente reduzir os seus custos de operação; a estratégia de diferenciação, que é quando a empresa busca diferenciar o seu produto no mercado; e o enfoque, que ocorre quando a empresa aplica uma das duas estratégias anteriores a um subgrupo de clientes com características comuns, tais como a renda, a idade, a cultura, os estilos de vida, dentre outras.

\subsection{Estratégia de manufatura}

Pode-se dizer que o principal precursor do conceito de estratégia de manufatura é Wickham Skinner que ao final da década de 60 lança o artigo Manufacturing - missing link in corporate strategy (SKINNER, 1969) em que afirma que a manufatura deve elaborar uma política, ou estratégia, de modo a dar apoio à competitividade da empresa.

Em um artigo posterior Skinner (1974) afirma que a função manufatura deve construir um conjunto de habilidades ou objetivos que lhe permita alta competitividade. Com efeito, os objetivos de desempenho, assunto amplamente abordado na literatura (ex. GIANESI, 1998; SILVA e SANTOS, 2005; TRACEY et al, 1999), consistem naqueles aspectos que a manufatura deve atingir determinado nível de performance, de modo a contribuir para a competitividade da empresa. Segundo Slack (2002) os objetivos de desempenho são: qualidade, rapidez, pontualidade, custo e flexibilidade, que se divide em: flexibilidade de mix, que é a capacidade do sistema de produção em produzir ampla variedade de produtos; flexibilidade de produtos, que é a capacidade de produzir novos produtos e alterar os existentes; e flexibilidade de volume, que é a capacidade do sistema de produção em responder adequadamente às variações dos níveis de demanda.

Hill (1994) classifica os objetivos de desempenho em: ganhadores de pedido, que são aqueles que contribuem decisivamente para a realização do pedido do cliente; qualificadores, que são aqueles em que a manufatura deve possuir um patamar mínimo para que seu produto possa ser considerado como potencial escolha pelo cliente; e menos importantes, que contribuem pouco para a competitividade do produto. A lógica da estratégia de manufatura se baseia, portanto, em definir 
os objetivos de desempenho em consistência com a estratégia competitiva e demais estratégias funcionais, e posteriormente configurar os seus recursos. Estes recursos são classificados em estruturais, que são os elementos tangíveis do sistema de produção, e infra-estruturais, que são os elementos que embora intangíveis, definem o modo de funcionamento deste sistema.

Começando pelos recursos estruturais, a política de capacidade é classificada em três abordagens por Olhager et al (2001), são elas: a liderança, que ocorre quando a capacidade é adicionada antes dos aumentos de demanda; a defasagem, que consiste em adicionar capacidade somente quando o sistema de produção se encontra com alta taxa de utilização; e a política que busca conciliar as duas anteriores. Já a política de localização das instalações diz respeito à distribuição geográfica das operações de manufatura, que pode ser centralizada, ou descentralizada.

A integração vertical está relacionada às operações que a manufatura manterá sob a sua responsabilidade. Pode-se considerar também o caminho inverso, ou seja, quais operações serão delegadas para os agentes da cadeia produtiva. Quanto ao nível de automação empregado Maccarthy e Fernandes (2000) apresentam os seguintes tipos: normal, que compreende qualquer tipo de automação em que o ser humano possui alto grau de participação na operação; flexível, em que o controle da operação pelo computador é predominante; rígida, que trabalha com equipamento especializado e dedicado a tarefas específicas, sendo o processo totalmente automatizado; e mista, quando existem unidades produtivas com diferentes níveis de automação. Outro elemento importante a se considerar é o tipo de arranjo físico empregado; neste caso, os tipos mais comuns são: o posicional, o por processo, o celular e o por produto.

A configuração dos recursos infra-estruturais tem papel determinante no modo de funcionamento do sistema de produção. A gestão da mão-de-obra será considerada em termos dos métodos de recompensa e incentivo do trabalhador e da forma de organização do trabalho. Os métodos de incentivo se referem principalmente ao tipo de remuneração e progressão na carreira. No tocante à organização do trabalho Marx (1998) apresenta os seguintes tipos: o baseado na escola clássica, que tem como característica a especialização do trabalhador, baixo nível de autonomia, divisão dos trabalhadores entre aqueles que executam tarefas operacionais e de gestão (inspeção e supervisão das atividades); os grupos semi-autônomos, abordagem resultante da escola sociotécnica, em que os operários possuem alto nível de liberdade no que diz respeito à divisão de tarefas e à gestão do trabalho; e os grupos enriquecidos, em que o trabalho é realizado também em equipe, porém com menor nível de liberdade, se comparado com os grupos semi-autônomos.

O sistema de qualidade está relacionado às políticas da qualidade utilizadas no sistema de produção, tais como o controle da qualidade total, as certificações, a manutenção preventiva, as ferramentas da qualidade (controle estatístico do processo, inspeção, análise de processos, .....), assunto este amplamente abordado na literatura. Em relação ao sistema de Planejamento e Controle 
da Produção (PCP) deve-se definir qual abordagem utilizar - Materials Requirements Planning (MRP-I), Manufacturing Resource Planning (MRP-II), Just in Time (JIT), Optimized Production Technology (OPT), dentre outras, como pode ser observado na literatura.

A política de relacionamento com a cadeia de suprimentos tem recebido destaque de vários autores (ex. MAIA et al, 2005; SILVA; SANTOS, 2005) nos últimos anos. Isto se deve principalmente ao fato de que a performance em determinado objetivo de desempenho em grande parte é determinada pelas características das matérias-primas que a empresa adquire de seus fornecedores. Em relação a esta política pode-se verificar empresas que mantêm apenas o relacionamento de mercado com os agentes da cadeia produtiva, e empresas que buscam estreitar este relacionamento por meio do trabalho conjunto.

\section{Metodologia}

O objetivo deste artigo é analisar a relação entre estratégias competitivas e de manufatura em três manufaturas calçadistas de grande porte (com mais de 500 funcionários), situadas em Franca, interior de São Paulo. Em relação à estratégia competitiva é utilizado o modelo das estratégias genéricas de Porter (1986); são também analisados aspectos relativos à configuração das funções marketing e P\&D. Em relação à estratégia de manufatura serão analisados os aspectos relativos à configuração dos objetivos ganhadores de pedido e dos recursos estruturais e infraestruturais. $\mathrm{O}$ delineamento utilizado é o estudo de caso, que permitiu analisar em profundidade as manufaturas e daí extrair relações teóricas que visam contribuir para a literatura. A coleta de dados foi realizada por meio de entrevista com questões abertas e fechadas, aplicadas a profissionais da alta gestão das empresas.

A empresa A possui um total de 2100 funcionários se dedicando exclusivamente à fabricação de calçado masculino de alto padrão. Esta empresa possui várias unidades produtivas, sendo uma grande unidade no interior do Estado do Ceará, uma unidade na cidade de Franca, e outras unidades em cidades vizinhas a Franca, destinadas a realização de fases do processo intensivas em mão-de-obra. A empresa B possui apenas uma unidade na cidade de Franca, com aproximadamente 700 funcionários, esta empresa fabrica calçado masculino adulto e infantil de alto e médio padrão de qualidade. A empresa $C$ possui 600 funcionários, ela se dedica à fabricação de tênis de baixo padrão de qualidade, voltado ao atendimento da população de menor renda. As empresas A e B estão voltadas para os mercados interno e externo, a empresa C está voltada apenas para o mercado interno. 


\section{Apresentação dos Dados}

No quadro 1 são apresentados os aspectos das empresas pesquisadas quanto à configuração da estratégia competitiva e das funções marketing e P\&D. A empresa A enquadra-se na estratégia de diferenciação, pois ela possui um produto com projeto diferenciado, privilegiando a alta qualidade e a imagem de marca. Já a estratégia da empresa B está voltada para duas frentes, a principal é a dedicada à fabricação de um produto com alto padrão de qualidade, no que diz respeito ao conforto, sendo esta uma estratégia de diferenciação. A segunda consiste na fabricação de calçados de baixo preço, que chegam a custar metade ou até um terço do calçado de alto padrão; estes são baseados no uso de componentes de menor custo e menor qualidade, sendo esta uma estratégia de baixo custo. A empresa $\mathrm{C}$ fabrica tênis cujo preço para o consumidor final está bem abaixo daquele praticado pelas líderes do mercado, utilizando, portanto, a estratégia de baixo custo.

Nota-se que as empresas A e B realizam altos investimentos na consolidação da marca e da imagem, participando de feiras nacionais e internacionais para se interarem das tendências da moda e para a divulgação de seus produtos, além de utilizarem vários outros mecanismos de divulgação voltados para o consumidor. Já a empresa $\mathrm{C}$ realiza poucos investimentos em divulgação, sendo esta voltada apenas para o lojista. Esta empresa participa apenas de feiras nacionais como forma de divulgação de seus produtos.

As empresas A e B também realizam amplos investimentos na função P\&D possuindo ampla equipe de profissionais para geração do conceito (estilistas) e para a geração do protótipo dos produtos (modelistas). Os calçados destas empresas são baseados na moda européia. Já a empresa $\mathrm{C}$ possui uma estrutura de $P \& D$ bem enxuta, realizando poucos investimentos na geração do conceito de seus produtos, nesta função trabalha apenas um profissional modelista. Os seus produtos são baseados nos lançamentos das empresas líderes de seu segmento. 
Quadro 1 - Configuração da estratégia competitiva e das funções marketing e P\&D.

\begin{tabular}{|c|c|c|c|}
\hline & Empresa A & Empresa B & Empresa C \\
\hline $\begin{array}{l}\text { Estratégia } \\
\text { Competitiva }\end{array}$ & Diferenciação & Diferenciação e Baixo Custo & Baixo \\
\hline $\begin{array}{l}\text { Configuração } \\
\text { da Função } \\
\text { Marketing }\end{array}$ & $\begin{array}{l}\text { Participação de feiras } \\
\text { nacionais e internacionais; } \\
\text { Realização de campanhas na } \\
\text { mídia; } \\
\text { Alto investimento em } \\
\text { divulgação (outdors; revistas, } \\
\text { televisão .....) }\end{array}$ & $\begin{array}{l}\text { Participação de feiras } \\
\text { nacionais e internacionais; } \\
\text { Realização de campanhas na } \\
\text { mídia; } \\
\text { Alto investimento em } \\
\text { divulgação (outdors; revistas, } \\
\text { televisão .....) }\end{array}$ & $\begin{array}{l}\text { Participação de feiras } \\
\text { nacionais; } \\
\text { Baixo investimento em } \\
\text { divulgação. Esta é voltada } \\
\text { apenas para o lojista }\end{array}$ \\
\hline $\begin{array}{l}\text { Configuração } \\
\text { da Função } \\
\text { P\&D }\end{array}$ & $\begin{array}{l}\text { Produto baseado na moda } \\
\text { européia; } \\
\text { Estilista e modelista próprios }\end{array}$ & $\begin{array}{l}\text { Produto baseado na moda } \\
\text { européia; } \\
\text { Estilista e modelista próprios }\end{array}$ & $\begin{array}{l}\text { Modelista próprio; } \\
\text { Modelos baseados nas marcas } \\
\text { líderes do mercado }\end{array}$ \\
\hline
\end{tabular}

Fonte: $\mathrm{O}$ autor.

O quadro 2 apresenta os principais aspectos da configuração da estratégia de manufatura nas empresas. Quanto aos objetivos ganhadores a empresa A valoriza a qualidade do produto, significando neste caso alto nível de projeto (design), durabilidade e conforto. A empresa $\mathrm{B}$, na sua linha principal valoriza a qualidade, no que diz respeito principalmente à durabilidade e ao conforto do produto, já na sua linha de médio padrão ela valoriza principalmente o baixo custo, o que é conseguido graças ao uso de componentes de menor custo. A empresa $\mathrm{C}$ prioriza o baixo custo de produção.

Todas as empresas adicionam capacidade antes da total utilização dos ativos, o que configura a política de liderança de capacidade. No tocante à localização, a empresa A possui uma política de descentralização, tendo várias unidades produtivas. Neste caso o objetivo é obter menores custos provenientes de mão-de-obra mais barata e de incentivos fiscais das localidades onde elas estão.

A empresa A se dedica à produção do calçado, produzindo também uma parte do solado consumido. A empresa B possui uma política consciente de integração vertical, beneficiando e produzindo respectivamente todo o couro e solado. Já a empresa $\mathrm{C}$ adotou o caminho inverso, ela atualmente terceirizou a produção para uma holding de sete pequenas manufaturas, que operam sob a mesma planta industrial, organizadas num único sistema de produção, todos os insumos são comprados externamente sob a coordenação desta empresa.

A tecnologia destas três empresas deve ser considerada como mista, uma vez que utilizam alguns equipamentos de controle computadorizado, sendo, entretanto, a maioria dos equipamentos de controle mecânico. Todas as empresas utilizam o arranjo físico por produto, em que o calçado é montado em várias etapas, sendo durante este processo transportado numa esteira de montagem. 
Também são iguais os sistemas de recompensa e de organização do trabalho, o primeiro se baseia na remuneração fixa dos funcionários da esteira e na remuneração por produtividade de funcionários com alto nível de especialização em tarefas (tal como o cortador de couro). A organização do trabalho de todas as empresas é baseada nos princípios da escola clássica. As empresas A e B utilizam os círculos de controle da qualidade para resolução de problemas do chão de fábrica, a empresa $\mathrm{C}$ não utiliza nenhuma ferramenta de qualidade. Todas as empresas utilizam o sistema MRP II para as atividades de PCP.

Quadro 2 - Configuração dos elementos da estratégia de manufatura

\begin{tabular}{|c|c|c|c|}
\hline & Empresa A & Empresa B & Empresa C \\
\hline Objetivos ganhadores & $\begin{array}{l}\text { Qualidade (conforto, } \\
\text { durabilidade e design) }\end{array}$ & $\begin{array}{l}\text { Qualidade (Conforto e } \\
\text { durabilidade); Custo }\end{array}$ & Custo \\
\hline Política de capacidade & Liderança & Liderança & Liderança \\
\hline Localização de Instalações & Descentralizada & Centralizada & Centralizada \\
\hline $\begin{array}{ll}\text { Integração } & \text { vertical/ } \\
\text { desverticalização } & \end{array}$ & $\begin{array}{l}\text { Produz parte do solado } \\
\text { consumido }\end{array}$ & \begin{tabular}{|lr} 
Além da produção & de \\
calçado, ela integra & as \\
atividades & de \\
beneficiamento de couro \\
e produção de solado
\end{tabular} & $\begin{array}{l}\text { Terceirizou toda a } \\
\text { produção para empresas } \\
\text { que operam sob a mesma } \\
\text { planta industrial }\end{array}$ \\
\hline Tecnologia de processos & Mista & Mista & Mista \\
\hline Arranjo Físico & Por produto & Por produto & Por produto \\
\hline Sistema de recompensa & $\begin{array}{l}\text { Por produtividade } \\
\text { individual e fixo }\end{array}$ & $\begin{array}{l}\text { Por produtividade } \\
\text { individual e fixo }\end{array}$ & $\begin{array}{l}\text { Por produtividade } \\
\text { individual e fixo }\end{array}$ \\
\hline Organização do trabalho & $\begin{array}{l}\text { Baseado na escola } \\
\text { clássica }\end{array}$ & $\begin{array}{l}\text { Baseado na escola } \\
\text { clássica }\end{array}$ & $\begin{array}{l}\text { Baseado na escola } \\
\text { clássica }\end{array}$ \\
\hline Sistema de qualidade & $\begin{array}{l}\text { Círculos de controle da } \\
\text { qualidade }\end{array}$ & $\begin{array}{l}\text { Círculos de controle da } \\
\text { qualidade }\end{array}$ & \\
\hline Sistema de PCP & MRP II & MRP II & MRP II \\
\hline $\begin{array}{l}\text { Relacionamento com a } \\
\text { cadeia de suprimentos }\end{array}$ & $\begin{array}{l}\text { Definição conjunta de } \\
\text { componentes } \\
\text { supervisão no } \\
\text { beneficiamento do couro }\end{array}$ & $\begin{array}{l}\text { Controle total na } \\
\text { produção do couro e no } \\
\text { beneficiamento de solado }\end{array}$ & $\begin{array}{l}\text { Relacionamento de } \\
\text { mercado com os } \\
\text { fornecedores e alto poder } \\
\text { de controle sobre os } \\
\text { prestadores de serviço }\end{array}$ \\
\hline
\end{tabular}

Fonte: $\mathrm{O}$ autor

Em relação ao relacionamento com a cadeia produtiva a empresa A possui forte poder de influência sobre os fornecedores de solado e de couro. Ela realiza em parceria com os primeiros o projeto de solados diferenciados, em que possui exclusividade de compra. Já em relação aos curtidores de couro esta empresa acompanha todo o processo de beneficiamento, desde o abate do 
gado até sua fase final, possuindo nestes agentes supervisores da qualidade. A empresa B possui total controle sobre a produção de solado e couro, visto que integrou verticalmente estas atividades, definindo componentes com características exclusivas. A empresa $\mathrm{C}$ utiliza seu grande poder de compra para conseguir economias de escala pecuniárias, possuindo com seus fornecedores o relacionamento de mercado.

\section{Análise dos Resultados}

É interessante ressaltar que todas as empresas possuem planejamento estratégico formalizado, nota-se nos três casos estudados forte consistência entre a configuração da estratégia competitiva e a configuração das funções marketing e P\&D. Enquanto as empresas A e B, que focam suas principais linhas de produtos na diferenciação, realizam fortes investimentos em diversos mecanismos de divulgação e no seu projeto, a empresa $\mathrm{C}$, que tem no baixo custo o seu objetivo principal, possui as estruturas de marketing e P\&D enxutas, realizando o mínimo de investimento possível. É interessante lembrar que a empresa B também produz calçados de médio padrão como forma de melhor utilização da capacidade.

Ocorre do mesmo modo forte consistência entre a estratégia competitiva e objetivos ganhadores de pedido das empresas. Entretanto, nota-se que diferentes objetivos de desempenho, não necessariamente implicam em diferentes configurações dos recursos produtivos. Isto fica evidente no fato de que embora priorizem diferentes objetivos de desempenho as empresas configuram da mesma forma a política de capacidade, a tecnologia de processos, o arranjo físico, o sistema de recompensa, a organização do trabalho e o sistema de PCP. Esta situação certamente é favorecida por questões como: influência cultural do arranjo produtivo de Franca; a facilidade de acesso à tecnologia, o que permite que todas as empresas utilizem o sistema MRP II e a tecnologia de processo mista; e a natureza do produto e de sua fabricação, o que justifica em parte o mesmo tipo de arranjo físico.

Por outro lado, nota-se que o fato de uma empresa priorizar determinado objetivo de desempenho não faz com que ela deixe de dar atenção a outros. Isto pode ser notado nas estratégias de descentralização das unidades produtivas e integração vertical realizadas respectivamente pelas empresas A e B. O motivo principal para estas abordagens segundo os profissionais entrevistados é a redução de custos de produção, o que lhes permitem maior competitividade nos mercados em que atuam.

A empresa C consegue implementar sua estratégia de baixo custo terceirizando completamente sua produção para empresas de pequeno porte; este tipo de abordagem visa diminuir as responsabilidades diretas sobre a produção, bem como aproveitar incentivos da legislação para as 
empresas de pequeno porte. Notam-se nos três casos que as empresas buscam o mesmo objetivo, o baixo custo, por meio de estratégias completamente diferentes.

Por fim, é interessante ressaltar o importante papel da cadeia produtiva na conquista dos objetivos de desempenho. Isso ocorre tanto com as empresas que buscam qualidade, como com as que buscam baixo custo. A qualidade é conseguida graças ao forte poder de influência sobre a cadeia, no que diz respeito à inspeção de operações dos fornecedores e desenvolvimento conjunto de componentes, o que ocorre com as empresas A e B. Enquanto que o baixo custo se consegue simplesmente pela compra de matérias-primas de menor valor agregado, que substituem as de alto valor, o que ocorre com as empresas B(para os produtos de médio padrão) e C. A importância dos insumos na estratégia de manufatura fica evidente no caso da empresa B que produz calçados de alto e médio padrão. Neste caso a diferença entre estes produtos está principalmente na qualidade dos componentes utilizados.

\section{Conclusões}

Observou-se nas empresas que diferentes estratégias competitivas levam a diferentes configurações das funções marketing e $\mathrm{P} \& \mathrm{D}$, o que comprova o raciocínio normalmente desenvolvido na literatura. Quanto à estratégia de manufatura nota-se o seguinte paradoxo: vários dos seus elementos foram configurados da mesma forma em empresas que priorizam diferentes objetivos de desempenho, por outro lado, existem diferentes configurações de um determinado recurso (descentralização de unidades/integração vertical/terceirização) na busca do mesmo objetivo. Este paradoxo mostra que existem fatores externos à estratégia de manufatura que são capazes de influenciá-la, como é o caso da cultura empresarial, natureza do produto, dentre outros. Mostra também que existem diferentes formas de se buscar o mesmo objetivo, uma vez que não fica evidente uma solução que seja superior a todas as demais.

Outro aspecto a ser considerado é que o processo de produção das empresas é similar, o que torna o seu desempenho bastante equilibrado neste quesito. Isso faz com que o relacionamento com a cadeia produtiva se torne um elemento essencial, seja na busca do baixo custo, ou da diferenciação.

Esta pesquisa contribui para a literatura à medida que apresenta uma visão empírica de como a estratégia de manufatura e a configuração de aspectos das funções marketing e P\&D servem como meios efetivos para a concretização da estratégia competitiva das empresas. Ela também mostra nuances práticas que não estão presentes na literatura, portanto complementando-a. 


\begin{abstract}
This paper analyses the relationship between competitive and operations strategy in footwear manufactories. There were visited three large firms (with more than 500 employees) located in Franca, in São Paulo State. The main conclusion is that the competitive strategy configuration has deep influence in the marketing and research \& development (R\&D) configuration. However, considering the manufactory function, one specific competitive strategy not necessarily determines only one way to configure its elements. Independently of the strategy that was chosen, the firms always pay special attention to the costs.

Key -Words: Competitive Strategy, Operations Strategy, Footwear Industry.
\end{abstract}

\title{
Referências
}

ABICALÇADOS. Disponível em: <"http://www.abicalcados.com.br">. Acessado na seção principal em: 18/07/2003.

ANDERSON, P. Barreiras não-tarifárias às exportações brasileiras no Mercosul: o caso de calçados. Rio de Janeiro: IPEA, 2001. Disponível em: <http://www.ipea.gov.br/pub/td/td_2001/td0791.pdf >. Acesso em: 13/09/2005.

ANSOFF, I. Corporate Strategy. New York: MC Graw-Hill, 1965.

GAVETti, G.; LEVINTHAL, D. A. The Strategy Field from Perspective of Management Science: Divergent Strands and Possible Itegration. Management Science, v.50, n.10, p.1309-1318, 2004.

crossef

GIANESI, I. G. N. Implementing manufacturing strategy through production planning. International Journal of Operations and Production Management, v.18, n.3, p.286-299, 1998.

cross ${ }^{\text {ref }}$

HAYES, R. H. WHEELWRIGHT, S. C. Restoring our competitive edge: competing through manufacturing. New York: John Wiley, 1984.

HILL, T. Manufactoring strategy: text and cases. $2^{\text {nd }}$ ed., Burr Ridge: Irwin, 1994.

MACCARTHY, B. L.; FERNANDES, F. C. F. A multi-dimensional classification of production systems for the design and selection of production planning and control systems. Production Planning and Control, v.11, n.5, p.481496, 2000.

cross ${ }^{\text {ref }}$

MAIA, J. L.; CERRA, A. L.; ALVES FILHO, A. G. Inter-relações entre estratégia de operações e gestão da cadeia de suprimentos: estudos de caso no segmento de motores para automóveis. Gestão \& Produção, v.12, n.3, p.377-391, set-dez, 2005.

MARX, R. Trabalho em grupos e autonomia como instrumentos de competição. São Paulo: Atlas, 1998.

MinTZBERG, H. AHLSTRAND, B. LAMPEL, J. Safári de estratégia: Um roteiro pela selva do planejamento estratégico. Porto Alegre, Bookman, 2000.

OLHAGER, J.; RUDBERG, M.; WIKER, J. Long-term capacity management: Linking the perspectives from manufacturing strategy and sales and operations planning. International of production economics, v.69, p.215-225, 2001 .

PORTER, M. Estratégia competitiva: técnicas para análise da indústria e concorrência. $26^{\text {a }}$ edição, Rio de Janeiro: Campus, 1986.

SCHMITZ, H. Global competition and local cooperation: Success and failure in the Sinos Valley, Brazil. World Development, v.27, n.9, p.1627-1650, 1999. 
SLACK, N. Vantagem competitiva em manufatura: atingindo a competitividade nas operações industriais. São Paulo: Atlas, 2002.

SEBRAE. Seção Estudos e Pesquisas. Disponível em: www.sebrae.com.br. Acesso em 15/01/2007.

SILVA, E. M.; SANTOS, F. C. A. Análise do alinhamento da estratégia de produção com a estratégia competitiva na indústria moveleira. Revista Produção, v.15, n.2, p.286-299, maio-agosto, 2005.

SKINNER, W. Manufacturing - missing link in corporate strategy. Harvard Business Review, p.136-144, may-june, 1969.

The focused factory. Harvard Business Review, p.113-121, may-june, 1974.

SUN, H.; HONG, C. The alignment between manufacturing and business strategies: its influence on business performance. Technovation, v.22, p.699-705, 2002.

cross'ref

TRACEY, M.; VONDEREMBSE, M. A.; LIM, J. S. Manufacturing technology and strategy formulation: keys to enhancing competitiveness and improving performance. Journal of Operations Management, v.17, p.411-428, 1999.

cross ${ }^{\text {ref }}$

WHITTINGTON, R. O que é estratégia. São Paulo: Thomson, 2002.

\section{Dados dos autores:}

Nome completo: Sergio Evangelista Silva

Doutorando em Engenharia de Produção UFSCar

Professor Assistente na Universidade do Estado de Minas Gerais - FESP-

UEMG, Campus de Passos, Minas Gerais.

Faculdades de Informática e de Administração de Passos

Endereço completo: Av. Juca Stockler, 1130, Bairro Belo Horizonte, Passos, MG, Brasil.

CEP - 37900-10635

Telefones: Tel. 35-3529-8013

E-mail: silvaevangelista@passosuemg.br

Nome completo: Dr. Flávio César Faria Fernandes

Filiação institucional: UFSCar

Departamento de Engenharia de Produção

Função ou cargo ocupado: Professor Associado

Endereço Completo: Rodovia Whashington Luiz, km, 235

Departamento de Engenharia de Produção DEP/UFSCar São Carlos, SP,

Brasil, CEP 13565905,

Telefone: (16) 3351-8236. r.9214

E-mail: dfcf@power.ufscar.br 
Recebido para publicação em: 12/11/2007

Aceito para publicação em: 26/11/2007 